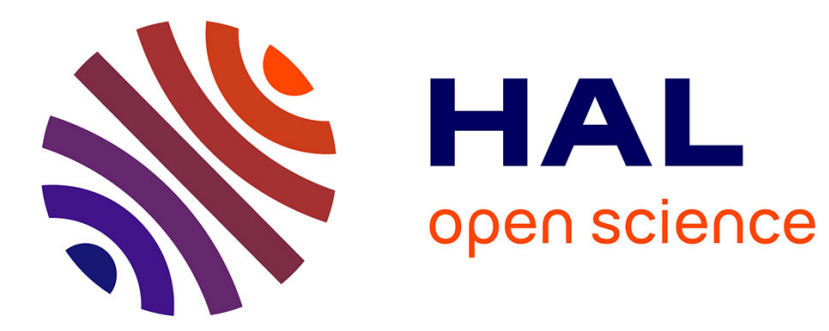

\title{
Third-order spectral phase compensation in parabolic pulse compression
}

Yoann Zaouter, Dimitris N. Papadopoulos, Marc Hanna, Frédéric Druon, Eric Cormier, Patrick Georges

\section{- To cite this version:}

Yoann Zaouter, Dimitris N. Papadopoulos, Marc Hanna, Frédéric Druon, Eric Cormier, et al.. Thirdorder spectral phase compensation in parabolic pulse compression. Optics Express, 2007, 15 (15), pp.9372-9377. hal-00700681

\section{HAL Id: hal-00700681 \\ https://hal-iogs.archives-ouvertes.fr/hal-00700681}

Submitted on 23 May 2012

HAL is a multi-disciplinary open access archive for the deposit and dissemination of scientific research documents, whether they are published or not. The documents may come from teaching and research institutions in France or abroad, or from public or private research centers.
L'archive ouverte pluridisciplinaire HAL, est destinée au dépôt et à la diffusion de documents scientifiques de niveau recherche, publiés ou non, émanant des établissements d'enseignement et de recherche français ou étrangers, des laboratoires publics ou privés. 


\title{
Third-order spectral phase compensation in parabolic pulse compression
}

\author{
Y. Zaouter ${ }^{1,2}$, D. N. Papadopoulos ${ }^{3}$, M. Hanna ${ }^{3}$, F. Druon ${ }^{3}$, E. Cormier ${ }^{2}$, P. Georges ${ }^{3}$ \\ ${ }^{1}$ Centre Lasers Intenses et Applications, CNRS - Université de Bordeaux 1 - CEA \\ 351 cours de la Libération, 33405 Talence, France \\ ${ }^{2}$ Amplitude Systemes, 6 allée du Doyen Georges Brus, 33600 Pessac, France \\ ${ }^{3}$ Laboratoire Charles Fabry de l'Institut d'Optique, CNRS, Univ Paris-Sud, RD 12891127 Palaiseau, France
}

zaouter@celia.u-bordeauxl.fr

\begin{abstract}
We report on the use of a hybrid compressor system consisting in a set of prisms embedded in a sequence of gratings specifically designed to optimize the compression of amplified parabolic pulses. Spectral phase measurements confirm that, as opposed to traditional gratings compressors, the third-order spectral phase is efficiently compensated in our system. We show that compression optimization leads to the enhancement of the temporal Strehl ratio from 0.67 using conventional compressor to 0.96 using our system. We demonstrate that prisms embedded gratings compressors assert itself as a simple and efficient solution to third-order dispersion compensation as this issue grows rapidly with the scaling of the performance in energy and bandwidth of parabolic fiber amplifiers.
\end{abstract}

C2007 Optical Society of America

OCIS codes: (320.5520) Pulse compression; (060.2320) Fiber optics amplifiers and oscillators; (060.5530) Pulse propagation and solitons; (060.7140) Ultrafast processes in fibers.

\section{References and links}

1. M. E. Fernman, V. I. Kruglov, B. C. Thomsen, J. M. Dudley, and J. D. Harvey, "Self-similar propagation and amplification of parabolic pulses in optical fibers," Phys. Rev. Lett. 84, 6010 (2000)

2. V. I. Kruglov, A. C. Peacock, J. M. Dudley, and J. D. Harvey, "Self-similar propagation of high-power parabolic pulses in optical fiber amplifiers," Opt. Lett. 25, 1753 (2000)

3. J. Limpert, T. Schreiber, T. Clausnitzer, K. Zöllner, H.-J. Fuchs, E.-B. Kley, H. Zellner, A. Tünnermann, "High-power femtosecond Yb-doped fiber amplifier," Opt. Express 10, 628 (2002)

4. G. Chang, A. Galvanauskas, H. G. Winful, and T. B. Norris, "Dependence of parabolic pulse amplification on stimulated Raman scattering and gain bandwidth," Opt. Lett. 29, 2647 (2004)

5. D. B. S. Soh, J. Nilsson, and A. B. Grudinin, "Efficient femtosecond pulse generation using a parabolic amplifier combined with a pulse compressor. I. Stimulated Raman-scattering effects," J. Opt.Soc. Am. B. 23, 10, (2006)

6. P. Tournois, "New diffraction grating pair with very linear dispersion for laser pulse compression," Electron. Lett. 29, 1414, (1993)

7. D. J. Kane, and R. Tebino, "Characterization of arbitrary femtosecond pulses using frequency-resolved optical gating," IEEE J. Quantum Electron. 29, 571 (1993)

8. S. Backus, C. G. Durfee, M. M. Murnane, H. C. Kapteyn, "High power ultrafast lasers," Rev. Sci. Instrum. 69, 1207 (1998)

9. A. Galvanauskas, "Ultrashort-pulse fiber amplifiers", in "Ultrafast lasers" CRC, p. 209 (2002)

10. L. Shah, Z. Liu, I. Hartl, G. Imeshev, G. C. Cho, and M. E. Fermann, "High energy femtosecond Yb cubicon fiber amplifier," Opt. Express.13, 4717 (2005).

11. S. Kane, J. Squier, J. V. Rudd, and G. Mourou, "Hybrid grating-prism stretcher-compressor system with cubic phase and wavelength tunability and decreased alignement sensitivity," Opt. Lett. 19, 1876 (1994)

12. S. Kane, and J. Squier, "Grism-pair stretcher-compressor system for simultaneous second- and third-order dispersion compensation in chirped-pulse amplification,"J. Opt. Soc. Am. B. 14, 661 (1997)

13. E. A Gibson, D. Gaudiosi, H. Kapteyn, R. Jimenez, S. Kane, R. Huff, C. Durfee, and S. Squier, "Efficient reflection grisms for pulse compression and dispersion compensation of femtosecond pulses," Opt. Lett. 31, 3363 (2006)

14. J. R. Buckley, S. W. Clark, and F. W. Wise, "Generation of ten-cycle pulses from an ytterbium fiber laser with cubic phase compensation," Opt. Lett. 31, 1340 (2006) 
15. L. Kuznetsova, F. W. Wise, S. Kane, J. Squier, "Chirped-pulse amplification of femtosecond pulses in a $\mathrm{Yb}$-doped fiber amplifier near the gain narrowing limit using a reflection grism compressor," presented at Advanced Solid-State Photonics, Vancouver, paper TuB3 (2007)

16. Y. Zaouter, J. Diderjean, F. Balembois, G. Lucas-Leclin, F. Druon, P. Georges, J. Petit, P. Golner, B. Viana, "47-fs diode pumped $\mathrm{Yb}^{3+}: \mathrm{CaGdAlO}_{4}$ laser ," Opt. Lett., 31, 119, (2006)

\section{Introduction}

Parabolic amplification has been demonstrated to be an elegant and efficient way to amplify femtosecond pulses in positive dispersion fibers [1-2]. Parabolic pulses are the asymptotic solution of the non-linear Schrödinger equation accounting for flat spectral gain, self-phase modulation (SPM) and group velocity dispersion (GVD). The interplay between SPM and GVD in presence of gain leads to purely linearly chirped amplified pulses. This linear chirp is in theory easily balanced and leads to an efficient recompression with conventional arrangement of optical elements (gratings or prisms) with negative GVD. Moreover, in the case of positive GVD fiber, a regime of wavebreaking-free propagation can be reached to overcome limit of pulse energy in classical regime [3]. However, this ideal asymptotic solution is experimentally inaccessible (or at least very limited) due to additional deleterious effects such as a limited gain bandwidth of the fiber, the higher dispersion orders, and stimulated Raman scattering (SRS).

Limitations in the parabolic regime have been investigated mainly taking into account the impact of SRS and finite gain bandwidth [4-5]. These works highlight the energy scaling aspects. Although observed and mentioned in several references [1,5], the impact of the presence of significant higher order spectral phase terms on the quality of the recompressed pulse has, to our knowledge, not been investigated in details. This effect is often not negligible since a remaining spectral phase degrades the temporal profile of the recompressed pulses. In parabolic amplification, the bandwidth increases along the fiber and is therefore related to the achievable gain. Since high-order dispersion effects - especially the third-order dispersion (TOD) - are particularly important for broad spectra, it is crucial to take them into account to understand and predict the recompressed pulse quality in parabolic amplifiers.

In this paper, we address the issue of optimal compression of parabolic pulses using a hybrid arrangement of embedded prisms in gratings pair [6]. The characterization of the remaining spectral phase of the parabolic pulses after compression is carried out using frequency-resolved optical gating (FROG) [7]. The TOD is identified to be the major source of pulse quality degradation when a standard gratings compressor is used. The hybrid arrangement of gratings and prisms allows the almost complete suppression of third-order dispersion, leading to an enhancement of both the time shortness and peak power of the recompressed pulses.

\section{Origin of the third-order spectral phase and compressor design}

The origin of third-order spectral phase in the recompressed pulse is multiple. First, the fiber dispersion is not pure GVD and introduces a non negligible TOD term. For typical large mode area fibers, the dispersion is dominated by the material, resulting in a third-order propagation constant of $\beta_{3}=6 \times 10^{-5} \mathrm{ps}^{3} \cdot \mathrm{m}^{-1}$. This contribution to the third order spectral phase solely depends on the fiber length $\mathrm{z}$. For our experimental parameters, we find $\varphi_{3}=24 \times 10^{-5} \mathrm{ps}^{3}$.

The second source of TOD is due to the gratings compressor geometry that adds to the third-order phase. For the asymptotic parabolic pulse, the second-order phase that must be compensated can be derived using the method of stationary phase, and is given by $\varphi_{2}=3 \beta_{2} / g$, where $\beta_{2}$ is the group velocity dispersion and $\mathrm{g}$ is the gain of the amplifying fiber. If we consider a standard gratings-based compressor, the ratio $\varphi_{3} / \varphi_{2}$ is fixed by the gratings characteristics, and is given by [8] 


$$
\frac{\varphi_{3}}{\varphi_{2}}=-\frac{3 \lambda}{2 \pi c}\left(1+\frac{\frac{\lambda}{d}\left(\frac{\lambda}{d}-\sin \gamma\right)}{1-\left(\frac{\lambda}{d}-\sin \gamma\right)^{2}},\right.
$$

where $\mathrm{d}$ is the grating period, $\lambda$ is the operating wavelength, $\mathrm{c}$ is the speed of light in vacuum, and $\gamma$ is the incidence angle on the first grating. A well known drawback of using gratings compressor to compress fiber-amplified pulses is that the ratio $\varphi_{3} / \varphi_{2}$ has an opposite sign compared to silica, resulting in an increase of the TOD in the compressed pulse. In our experiment, the gratings compressor induces $\varphi_{3}=28.6 \times 10^{-5} \mathrm{ps}^{3}$. The contributions from the fiber and the gratings compressor are therefore approximately equivalent.

The third source of TOD originates from SPM of spectrally asymmetric pulses. In fact, in the strongly stretched pulse regime, the accumulated SPM can be seen, in a first approximation, as a spectral phase proportional to the spectral intensity [9]. For ideal parabolic pulses, the spectrum is symmetric, and this effect is negligible. However, if the spectrum is not symmetric, for instance due to the fiber spectral gain profile, SPM results in an asymmetric phase. This effect can be used to compensate the other TOD sources [10]. However, this compensation scheme is power dependent. Overall, this contribution to TOD is hard to evaluate in the general case since it is dependent on the precise spectral shape evolution inside the amplifier. This spectral shape can vary depending on the input spectrum, the position of the central wavelength with respect to the gain spectral shape, higher-order effects that affect the spectrum, and so on. In the experiment described hereafter, the measured output spectrum is roughly symmetric, so that we expect this source of TOD to be small compared to the other contributions.

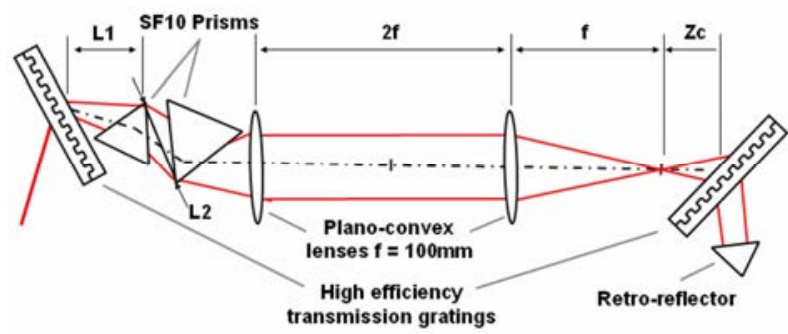

Fig. 1. Scheme of the hybrid gratings - prisms arrangement called grism used in the experiment. Both gratings have a line density of $1250-\mathrm{line} / \mathrm{mm}$.

Since the gratings compressor is a major source of TOD, we implemented a hybrid prismcompressor geometry allowing an independent adjustment of $\varphi_{2}$ and $\varphi_{3}$. Initially such a hybrid gratings - prims sequence compressor, known as "grism" and based on an idea of Tournois [6], has been used in Ti:sapphire-based chirp pulse amplifier to compensate simultaneously second and third-order dispersions in different CPA configurations [8,11,12]. Recently, grisms have been implemented in a femtosecond fiber oscillator leading to a record 33 -fs pulse generation [13], and in a fiber chirped-pulse amplification system [14]. Here, this geometry is used in the context of parabolic amplifiers. Figure 1 shows a sketch of the grism configuration used in the experiment. The dispersion abilities of the two prisms arrangement is heightened by its incorporation into the gratings compressor. Thus, the adjustment of the distance $\mathrm{Zc}$ between the second grating and the image of the first one sets the rough compression of the parabolic pulses, while a careful choice of the distance L between the apexes of the two prisms adds the necessary TOD for a global optimal compression. Simulations based on optical path calculations in this system showed that the $\varphi_{3} / \varphi_{2}$ ratio can 
be adjusted from the standard negative value of gratings compressor when $\mathrm{L}_{2}=0$ to positive values when $\mathrm{L}_{2}$ increases. With the gratings and prisms used in the experiment, this ratio can be tuned from $-4.2 \mathrm{fs}$ for $\mathrm{L}_{2}=0$ to $4.7 \mathrm{fs}$ for large $\mathrm{L}_{2}$. If the nonlinear part of the spectral phase can be neglected, the optimal value of the $\varphi_{3} / \varphi_{2}$ ratio for an ideal parabolic amplifier is given by

$$
\frac{\varphi_{3}}{\varphi_{2}}=\frac{\beta_{3} g z}{3 \beta_{2}} .
$$

In our experiment, this optimal ratio is equal to $3.5 \mathrm{fs}$, which is attainable using the hybrid gratings / prisms architecture.

\section{Experimental results}

The amplifier setup (see Fig. 2) consists in a 4 m-long double-clad ytterbium-doped large mode area (LMA) fiber with core diameter of $25 \mu \mathrm{m}$ and clad diameter of $250 \mu \mathrm{m}$ from Nufern. Both ends of the fiber are angle-cleaved at $8^{\circ}$ to suppress parasitic lasing. The fiber exhibits a pump absorption of $5.5 \mathrm{~dB} / \mathrm{m}$ at $976 \mathrm{~nm}$ This fiber is seeded by a femtosecond bulk oscillator based on a diode-pumped ytterbium-doped $\mathrm{CaGdAlO}_{4}$ crystal [15] providing 87-fs pulses at $1050 \mathrm{~nm}$ with an output power of $250 \mathrm{~mW}$ at a repetition rate of $105 \mathrm{MHz}$. The spectral bandwidth of the oscillator is $14.6 \mathrm{~nm}$ leading to time-bandwidth product (TBP) of 0.35 . The oscillator and amplification stage are isolated by an optical isolator and $50 \mathrm{~mW}$ of average power is seeded into the high-power fiber amplifier. These input pulses are sufficiently close to the optimum parabolic pulse amplification parameters for the output pulse spectrum and temporal profile to converge to a parabolic shape even with the short fiber length.

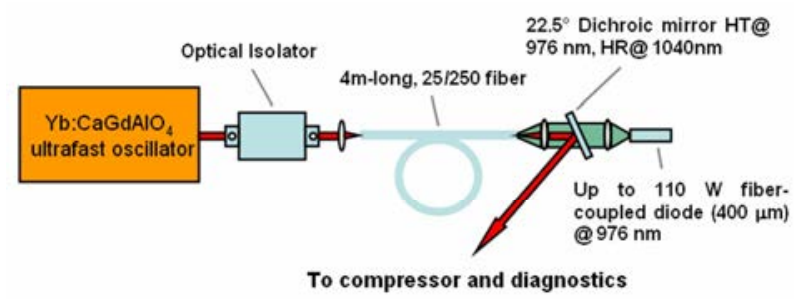

Fig. 2. Experimental setup of the parabolic pulse amplifier.

At $4 \mathrm{~W}$ output power, the pulse spectrum is broadened to $23 \mathrm{~nm}$ and the pulse duration extended to $2.2 \mathrm{ps}$ (TBP of 14) due to nonlinearities, gain and dispersion in the fiber. The parabolic character of the output is clearly seen on Figure 3 where we plot the intensity and phase, in both the spectral and temporal domain, retrieved from a second harmonic generation frequency-resolved optical gating (SHG FROG) measurement setup. In a first step, the pulses are compressed with a conventional setup consisting in a high-efficiency $1250-$ line/mm transmission-gratings compressor. The diffraction efficiency of these gratings is $93 \%$, leading to a theoretical compressor efficiency of $75 \%$. However, the experimental efficiency of the compressor was lower than expected i.e. close to $70 \%$ because of the depolarization of the output pulses which could not be entirely corrected by a set of wave plates located at the output of the amplifier. Additionally, we could not use the standard parallel gratings compressor configuration as the chirp accumulated during the amplification required a distance between the gratings of less than $10 \mathrm{~mm}$. Such a small separation could not be achieved due to the thickness of the substrates of the gratings. We thus have changed the configuration to an anti-parallel gratings arrangement similar to conventional grating stretchers. The distance between the second grating and the image of the first one through the optical system is, however, set negative causing this configuration to add negative GVD and act as a compressor. For optimal compression, significant wings show up in the FROG measurement, as shown in Fig. 4. The compressed pulse duration is $127 \mathrm{fs}$ corresponding to a 
TBP of 0.78. As expected, a significant cubic spectral phase remains, leading to important post- and pre-pulses as high as $13 \%$ of the main peak intensity. Alternatively, the temporal Strehl ratio (defined as the ratio between the actual peak power and the peak power obtained assuming a flat spectral phase) is in this case 0.67 .
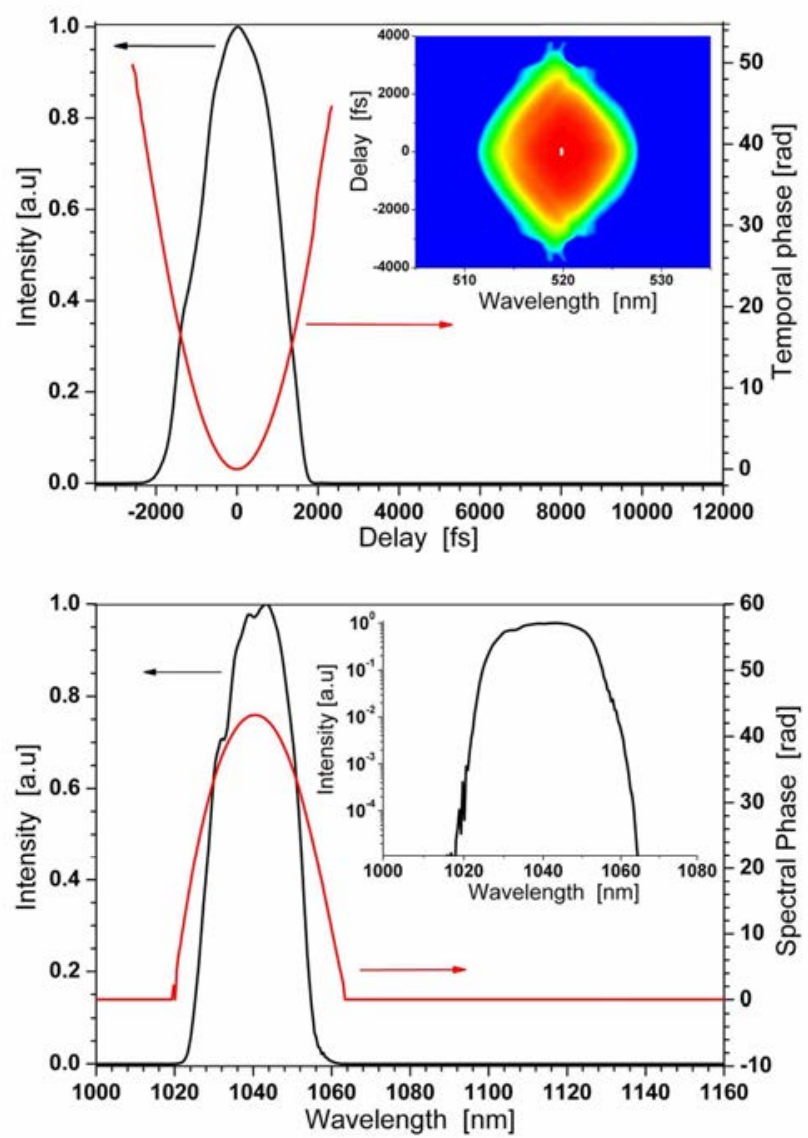

Fig. 3. Top: retrieved temporal intensity of parabolic pulses at $4 \mathrm{~W}$ output power from FROG measurement (inset). Bottom: retrieved spectral intensity (inset in Log scale). The FROG error is $61.10-4$ on a $512 \times 512$ grid (colors are in linear scale).

In order to get rid of the remaining cubic spectral phase, we have, in a second step, introduced two SF10 prisms within the two gratings sequence. Figure 4 shows the retrieved temporal intensity, spectral phase and FROG trace with the first prism placed at $\mathrm{L} 1=52 \mathrm{~mm}$ of the first grating, the two apexes of the SF10 prisms separated by L2 $=25 \mathrm{~mm}$ and the optical distance between the first grating and the first lens set to $\mathrm{f}=100 \mathrm{~mm}$. The cubic spectral phase and thus the temporal post-pulses are removed successfully and the temporal Strehl ratio has risen in this case to 0.96. Furthermore, the duration of the pulse is reduced down to $107 \mathrm{fs}$ corresponding to an improved TBP of 0.68 . One can notice that even if the spectrum bandwidth increases during the parabolic amplification, the compressed pulses exhibit a longer duration than the initial input pulse. This is due to the reshaping process of the sech pulse towards a parabolic pulse which has a theoretical TBP of 0.73 . The obtained TBP of 0.68 is lower than the theoretical value because the pulses have not completely converged to a parabolic shape. Finally, as the prisms were introduced into the compressor at Brewster angle, the global efficiency of the grism compressor is still $65 \%$. 


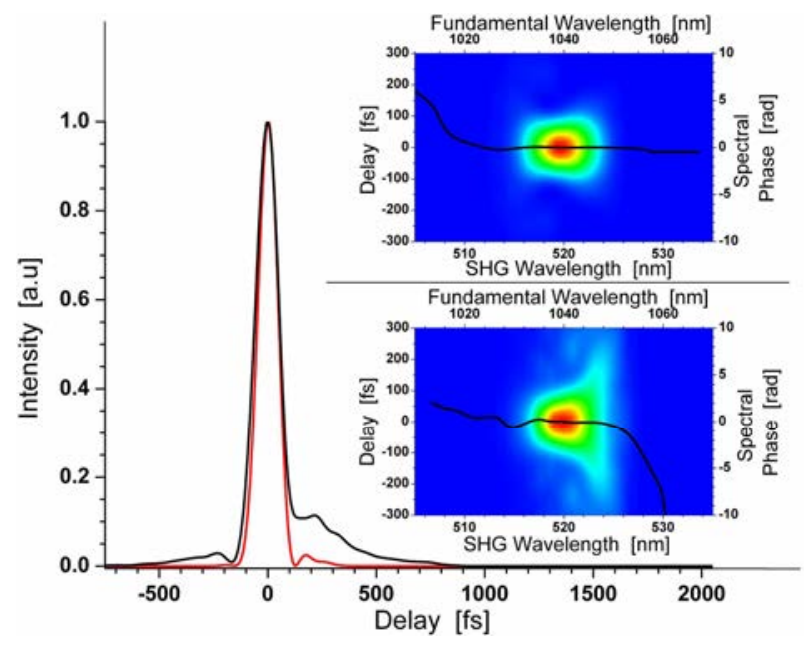

Fig. 4. Temporal intensities of the recompressed pulses with a grating arrangement (black curve) and its FROG trace (bottom inset) and with a grism arrangement (red curve) and its FROG trace (top inset). The FROG errors are $32.10^{-4}$ and $26.10^{-4}$ for the compression with gratings and with grism respectively, on $128 \times 128$ grids (colors are in linear scale).

\section{Conclusion}

In conclusion, we have investigated the third-order dispersion compensation in parabolic pulse compression and showed that the use of a hybrid gratings-prisms compressor enhances significantly the quality of the recompressed pulses compared to a conventional grating compressor. As an indicator, the temporal Strehl ratio has been raised from 0.67 for the grating compressor to 0.96 for the grism compressor. We have shown that remaining thirdorder spectral phase represents an important limitation in parabolic amplification of short pulses. It experimentally appears as the first limiting effect when the quality of recompressed pulses is considered. Moreover, this effect becomes increasingly important as the bandwidth of the spectra increases, and therefore must be addressed when scaling the output energy.

\section{Acknowledgments}

The authors acknowledge the financial support of the Réseau des Technologies Femtoseconde, the Conseil Regional d'Aquitaine, the Laserlab consortium and the Agence National de la Recherche under program HIPOLYFF. D. N. Papadopoulos acknowledges the Conseil Regional d'Ile de France for the financial support of his post-doctoral position. 